\title{
Reinforcing the Translational Bridge: Realizing the True Promise of Research Innovations
}

\author{
Alexandra Thomas, MD, FACP, University of Iowa
}

$\mathrm{I}$

n 2005 Elias A. Zerhouni, then director of the National Institutes of Health published a seminal essay, "Translational and Clinical Science - Time for a New Vi-

Lsion" in the New England Journal of Medicine. ${ }^{1}$ This work challenged stakeholders in the scientific enterprise to consider new models to "translate the remarkable scientific innovations we are witnessing into health gains for the nation." Since then debate has continued regarding how best to realize the potential of true translational work. While gains have certainly been made, historic, structural and philosophic hurdles persist which prevent us from fully achieving this promise.

Clinical Faculty Perspective

With this background, I offer one perspective, that of a clinical faculty member at a large public institution who has also recently served as a leader of the elected faculty body at the University of Iowa, on areas we might target as we try to take bench-top discoveries to the bedside or the clinic. A significant portion of my professional time is spent on direct patient care. This indeed is what drives my goal of participating in the conversation of how we can provide ever-better care for patients with breast cancer and ultimately for all patients.

My personal research spans several areas. At my institution I lead federally funded clinical trials in my discipline of breast oncology. I also direct industry funded clinical trials and partner with laboratory investigators to bring investigator-initiated studies to the clinic. One study which is currently open for enrollment looks at the role of inhibiting the RET receptor in hormone receptor positive breast cancer. Other translational work includes work investigating novel agents to treat chemotherapy-induced neuropathy and studies looking at new circulating serum markers of breast cancer.

Research I direct involves a collaboration of faculty from the Colleges of Medicine, Pharmacology and Public Health with the objective of utilizing the SEER (Surveillance, Epidemiology, and End Results program of the National Cancer Institute) data and other data sources to address questions related to breast cancer treatment and outcomes. My personal work seeks to answer questions which directly pertain to our patients, and looks at issues that we find frustrating in the clinic. Finally, I initiated and serve as the principal investigator for the University of Iowa Breast Molecular Epidemiologic Resource, which is a prospective study of tissue and epidemiologic data for individuals who are at high risk for or are diagnosed with a proliferative disorder of breast tissue. As of March 2015 over 1,000 patients have enrolled in 
this registry. This has served as a resource for bench investigators looking at a variety of questions in breast cancer. Notable recent externally funded work includes examining obesity, inflammation and breast cancer and a study looking at the role of novel fusion transcripts in breast cancer.

There are many areas that we might, as a community, elect to focus on to truly harness the potential of translational research. With the lens that I bring to clinical research and the healthcare enterprise, I put forth three areas that we could invest in to more completely produce healthcare gains for society: reward all members on the bridge from the laboratory to the clinic, support and sustain women in science, engage society. We might further consider that we, at public universities in the Midwest, in certain ways are uniquely situated to act on these opportunities based on our rich traditions of community and collaboration.

Reward All Members on the Translational Bridge from the Laboratory to the Clinic: Since the 2005 statement by the National Institutes of Health Director, scientific leaders have worked to embrace this model. An entire body of literature has evolved on "team science" which focuses on collaboration from bench to bedside or clinic as well as in cross-disciplinary work and cross-institutional work. Some have shown that while such coordination can have a leadtime, it ultimately demonstrates higher productivity with regard to publications and inclusion of many co-authors. ${ }^{2}$ However meaningful opportunities for respect of all contributions still exist. Traditional hierarchies in education persist.
Some argue that clinical research or including clinicians in research dilutes the rigor of academic work.

However, fully including clinicians in investigations is critical on several increasingly important fronts. Fundamental research which is linked to applied research is more readily supported by the public. Importantly, engaging those delivering the products of bench top investigations to patients further provides the opportunity for clinicians to bring back to their laboratory colleagues the pertinent, unanswered questions. This two-way dialogue is vital to making contemporary medical research relevant. Opportunities to fortify this communication and value all members of the translational bridge include encouraging diverse research portfolios, reconsidering what is valued in promotion and tenure and revisiting how awards and leadership roles are distributed.

Support and Sustain Women in Science: Some might argue that women have made great advances in participating in the scientific enterprise. However, the barriers to fully including women remain. We really need to look no further than the very recent comments by Nobel Laureate Tim Hunt who stated, “...let me tell you about my trouble with girls...three things happen when they are in the lab. You fall in love with them, they fall in love with you, and when you criticize them, they cry." 3 In 2013 Nature dedicated an entire issue to the topic of women in science. This publication was dedicated to a long-time editor Maxine Clarke who for years was known for her high scientific standards and for asking, "Where are the women?" 
Supporting and sustaining women in science is critical, as ultimately unless women are equal partners, society will be deprived of this intellectual resource. A myriad of opportunities exist to fully include women in our work. Important advances would include rewarding women equally. Equal pay and comparable recognition with awards still needs to be attained. We also need to make science and the environments in which it is practiced comfortable for women. Do we need to feminize science? No. Perhaps more aptly we could allow practicing science to be feminine. I would argue that in breast oncology, I have found a discipline which can have a very feminine aspect to it - can we expand that to allow it as part of other fields, would that help lung cancer and colon cancer patients? What about research in technology fields? Finally, we could better understand career breaks for having a family (which also ultimately helps men on a variety of levels) and proportionally give credit as one young scientist recently describes. ${ }^{4}$ In this we are asking science to support families, which further ensures our sustainability and helps all stakeholders.

Engage Society: The eloquent Harvard scientist Stephen Jay Gould who could magnetically draw the public to science explained his skill once stating, "So many scientists think that once they figure it out, that's all they have to do, and writing it up is just a chore. I never saw it that way. Part of the art of any kind of total scholarship is to say it well." 5 Perhaps at no time has this ability to tell our story been more relevant than today, when funding is short and we need to en- gage society to garner support for the vital work of discovery. I am able to deliver novel life-saving therapies to women today that were not available just a few years ago. Perhaps our community should more regularly showcase these incredible successes in ways that speak to non-scientists? What about the other side of this story, when we use the societal resources we are given poorly. Some in academia have criticized the Golden Fleece award, arguing that to succeed we need to have failures. Perhaps this is true to some extent, however the days of funding, philanthropic or governmental, for clearly irrelevant research are over. To maintain credibility with society we must be excellent stewards of public and private resources. We must show society, in language they understand, that what they award us helps overcome disease, hunger, poverty and leads to a better quality of life.

In engaging society with our work, we have another opportunity to collaborate across the translational bridge. Scientists need to articulate the value of their work - but can also collaborate here with their clinical colleagues- who sit at the nexus with the public and directly understand the value of scientific advances. We should use resources wisely and with respect and take opportunities to showcase meaningful, transformative research innovations. This means when the media calls, embrace the chance to discuss our projects. While this is not innately comfortable for many of us, perhaps we should strive to make it more a part of our culture, especially at public universities. 


\section{Conclusion}

The prospect of better realizing research innovations at the forefront of science holds immense promise for improved health and social outcomes. As Dr. Zerhouni wrote, "We now aim to stimulate the development of a brighter vision for translational and clinical research, to ensure that these disciplines remain powerful engines of creativity." I have tried to outline several achievable, and also imperative opportunities to come closer to fully attaining this goal. Undoubtedly some barriers will need to be removed, as again Dr. Zerhouni outlined: "...the NIH has the responsibility to work toward dissolving the artificial barriers that inevitably spring up...We persevere in our determination to provide opportunities for the research community and to challenge the status quo in transformative ways." Certainly, the shared goals of better health outcomes and improved global quality of life held by all stakeholders in the research enterprise can help move us collectively toward this vision.

\section{References:}

1. Zerhouni EA. Translational and clinical science--time for a new vision. The New England journal of medicine. Oct 13 2005;353(15):1621-1623.

2. Hall KL, Stokols D, Stipelman BA, et al. Assessing the value of team science: a study comparing center- and investigator-initiated grants. Am. J. Prev. Med. Feb 2012;42(2):157-163.

3. Lecturer who revealed Sir Tim Hunt's 'sexist' comments says she has no regrets about costing the Nobel Prize winner his job. The Daily Mail. June 25, 2015.

4. Nicholson E. Accounting for career breaks. Science. May 15 2015;348(6236):830.

5. Green M. Stephen Jay Gould. People. Vol 25, June 2,1986. 